\title{
A randomized double-blind study of the short-time treatment of obese patients with nonalcoholic fatty liver disease with ursodeoxycholic acid
}

V.N. Santos ${ }^{1}$, V.P. Lanzoni ${ }^{3}$, J. Szejnfeld ${ }^{2}$, D. Shigueoka ${ }^{2}$ and E.R. Parise ${ }^{1}$

\author{
'Disciplina de Gastroenterologia, Departamento de Medicina, \\ and Departamentos de ${ }^{2}$ Diagnóstico por Imagem and \\ ${ }^{3}$ Patologia, Escola Paulista de Medicina, \\ Universidade Federal de São Paulo, São Paulo, SP, Brasil
}

\section{Correspondence \\ E.R. Parise \\ Departamento de Medicina EPM, UNIFESP \\ Rua Botucatu, 740, $2^{\circ}$ andar \\ 03411-900 São Paulo, SP \\ Brasil \\ Fax: +55-11-5549-1711 \\ E-mail: parise@gastro.epm.br \\ Publication supported by FAPESP.}

Received October 14, 2002

Accepted May 5, 2003

\begin{abstract}
In order to determine the effect of ursodeoxycholic acid on nonalcoholic fatty liver disease, 30 patients with body mass indices higher than 25, serum levels of alanine aminotransferase (ALT), aspartate aminotransferase (AST) or $\gamma$-glutamyltransferase $(\gamma$-GT) at least more than 1.5 times the upper limit of normality, and hepatic steatosis demonstrated by ultrasonography were randomized into two groups of 15 patients to receive placebo or $10 \mathrm{mg} \mathrm{kg}^{-1}$ day $^{-1}$ ursodeoxycholic acid for three months. Abdominal computed tomography was performed to quantify hepatic fat content, which was significantly correlated with histological grading of steatosis $\left(\mathrm{r}_{\mathrm{s}}=-0.83, \mathrm{P}<0.01\right)$. Patient body mass index remained stable for both groups throughout the study, but a significant reduction in mean ( \pm SEM) serum levels of ALT, AST and $\gamma$-GT was observed only in the treated group (ALT $=81.2 \pm 9.7$, $44.8 \pm 7.7,48.1 \pm 7.7$ and $52.2 \pm 6.3 \mathrm{IU} / 1$ at the beginning and after the first, second and third months, respectively, $\mathrm{N}=14, \mathrm{P}<0.05$ ). For the placebo group ALT values were $66.4 \pm 9.8,54.5 \pm 7,60 \pm 7.6$ and 43.7 $\pm 5 \mathrm{IU} / \mathrm{l}$, respectively. No alterations in hepatic lipid content were observed in these patients by computed tomography examination $(50.2 \pm 4.2$ Hounsfield units (HU) at the beginning versus $51.1 \pm 4.1$ $\mathrm{HU}$ at the third month). These results show that ursodeoxycholic acid is able to reduce serum levels of hepatic enzymes in patients with nonalcoholic fatty liver disease, but this effect is not related to modifications in liver fat content.
\end{abstract}

\section{Introduction}

Nonalcoholic fatty liver disease (NAFLD) has been diagnosed with a higher frequency in the last years because of improved imaging methods. Most patients are asymptomatic and show no signs of disease $(1,2)$. Serum levels of hepatic enzymes (mainly ami-
Key words

- Nonalcoholic fatty liver disease

- Nonalcoholic steatohepatitis

- Fatty liver

- Ursodeoxycholic acid 
In spite of the high prevalence of hepatic steatosis and of the potential of nonalcoholic steatohepatitis (NASH) to progress to fibrosis and cirrhosis, no effective specific treatment is available. The procedure used today is based only on the removal of the possible causal factors $(5,6)$.

During the last few years ursodeoxycholic acid (UDCA) has been used to treat many diseases of the liver, such as hepatic cholestatic diseases and chronic hepatitis, among others (7-10). In uncontrolled studies, UDCA was used to treat NASH, with biochemical improvement of hepatic enzymes and of the inflammatory activity of the liver $(11,12)$.

In the present study we determined the effects of the administration of UDCA for three months on the serum levels of hepatic enzymes and on liver lipid content evaluated by CT and MRI in patients with NAFLD secondary to nonpathological obesity.

\section{Patients and Methods}

Patients with persistent abnormal levels of biochemical markers for more than six months, with a body mass index (BMI) higher than 25, alanine aminotransferase (ALT), aspartate aminotransferase (AST) or $\gamma$-GT levels at least more than 1.5 times the upper limit of normality, and ultrasonography showing signs of hepatic steatosis were enrolled in the study. Patients drinking more than 40 $\mathrm{g}$ ethanol per week, with decompensated diabetes mellitus, serum cholesterol and triglycerides above $300 \mathrm{mg} / \mathrm{dl}$, continuous intake of hepatotoxic medicines, positive hepatitis B surface antigen (HBsAg) and antihepatitis $\mathrm{C}$ virus (HCV) antibodies or other concomitant hepatic or recognized systemic diseases were excluded from the study.

Before the beginning of therapy and every month up to the end of treatment, patient BMI was calculated by the Quetelet method (13). All patients were tested for serum levels of ALT, AST, $\gamma$-GT, total cholesterol and triglycerides, thyroid-stimulating hormone, free T4 and glucose, HBsAg, and anti-HBc and anti-HCV antibodies (ELISA, Abbott Laboratories, North Chicago, IL, USA). MRI was performed with a Gyroscan S15 apparatus at 1.5 Tesla (Philips, Eindhoven, The Netherlands) and the Somaton DR scanner equipment (Siemens, Erlangen, Germany) was used for hepatic CT. Unenhanced scans were obtained at $125 \mathrm{kVp}, 230 \mathrm{mAs}$. Images were obtained using 8-mm collimation and $12-\mathrm{mm}$ increment. Liver attenuation values were obtained using a $2-\mathrm{cm}$ diameter circular region of interest, with the cursor placed at approximately the same site in each hepatic segment, avoiding artifacts or vessels. Attenuation measurements of the spleen were also made and the liver-to-spleen attenuation difference was calculated. Since CT studies could not be performed in patients with a body weight of more than $120 \mathrm{~kg}$, seven patients were not evaluated (three in the treated group and four in the placebo group).

Liver biopsy specimens were obtained by percutaneous puncture with Tru-Cut needles from all patients before starting treatment. Specimens were fixed in neutral buffered formalin, embedded in paraffin and stained with hematoxylin-eosin, Masson's trichrome and Perls' Prussian blue. The histopathological lesions were assessed and scored according to the degree of fat accumulation, portal and parenchymal inflammation, fibrosis, and ballooning degeneration. Macrovesicular steatosis was graded 0-3 based on the percentage of involved hepatocytes in the biopsy specimens (1: up to $33 \% ; 2$ : 33 to $66 \%$, and $3:>66 \%$ ) (14). NAFLD was classified into four types according to Matteoni et al. (1): type 1 - simple fatty liver, type 2 - fat plus inflammation, type 3 - fat with ballooning degeneration, and type 4 - fibrosis at any stage.

Patients were randomized into two groups of 15 patients to receive UDCA, $10 \mathrm{mg} \mathrm{kg}^{-1}$ day $^{-1}$ (divided into two daily doses), or a placebo administered in a double-blind man- 
ner for a period of three months. Monthly returns were scheduled for clinical and biochemical evaluation and for control of the use of medication. MRI and CT were performed at the beginning and at the end of the study.

The Human Studies Committee of the Federal University of São Paulo approved the study, and an informed consent was obtained from all patients.

\section{Statistical analysis}

Data are reported as means \pm SEM. The clinical, histological and biochemical pretreatment findings for the treated and placebo groups were compared by the unpaired $t$-test for continuous variables and by the $\chi^{2}$ test for comparison of frequency data. The mean values of the variables within the same group at different times were compared by analysis of variance for repeated measurements, complemented by the Bonferroni test for multiple comparisons. The paired $t$-test was applied to compare the values of CT attenuation before and at the end of treatment between control and treated patients. Spearman's correlation coefficient was calculated for the relationship between the attenuation values of CT or MRI and the histological grading of hepatic steatosis (15).

\section{Results}

The study groups were comparable regarding age, sex, associated factors, BMI, type of NAFLD and hepatic enzyme serum levels (Table 1). There was an association of obesity with dyslipidemia and diabetes in 12 patients.

No correlation was detected between conventional MRI and the histological grading for steatosis (Figure 1), while CT attenuation was inversely and significantly correlated with the degree of fatty liver accumulation $\left(r_{s}=-0.83, P<0.01 ;\right.$ Figure 2$)$.

During treatment, the patients' BMI re-
Table 1. Clinical and histological data for UDCA-treated and placebo groups of obese patients with nonalcoholic fatty liver disease.

\begin{tabular}{lcc}
\hline & UDCA & Placebo \\
\hline Age & $38.4 \pm 2.1$ & $36.6 \pm 3.1$ \\
Gender (male/female) & $14 / 1$ & $14 / 1$ \\
Etiology (obesity/dyslipidemia) & $15 / 1$ & $15 / 4$ \\
BMI (up to 29/more than 29) & $5 / 10$ & $7 / 8$ \\
NAFLD (types 1/2/3/4) & $6 / 4 / 2 / 3$ & $5 / 4 / 3 / 2$ \\
$\gamma$-GT (IU/I) & $100.7 \pm 23.7$ & $97.2 \pm 21.9$ \\
ALT (IU/I) & $79.8 \pm 9.21$ & $67.3 \pm 9.5$ \\
AST (IU/I) & $61.9 \pm 12.1$ & $40.5 \pm 9.9$
\end{tabular}

Data are reported as means \pm SEM for 15 patients in each group. ALT and AST: alanine and aspartate aminotransferase, respectively; BMI: body mass index; $\gamma$-GT: $\gamma$-glutamyltransferase; NAFLD: nonalcoholic fatty liver disease scored as described in Ref. 1; UDCA: ursodeoxycholic acid. No statistical differences were found for histological or clinical data between groups.

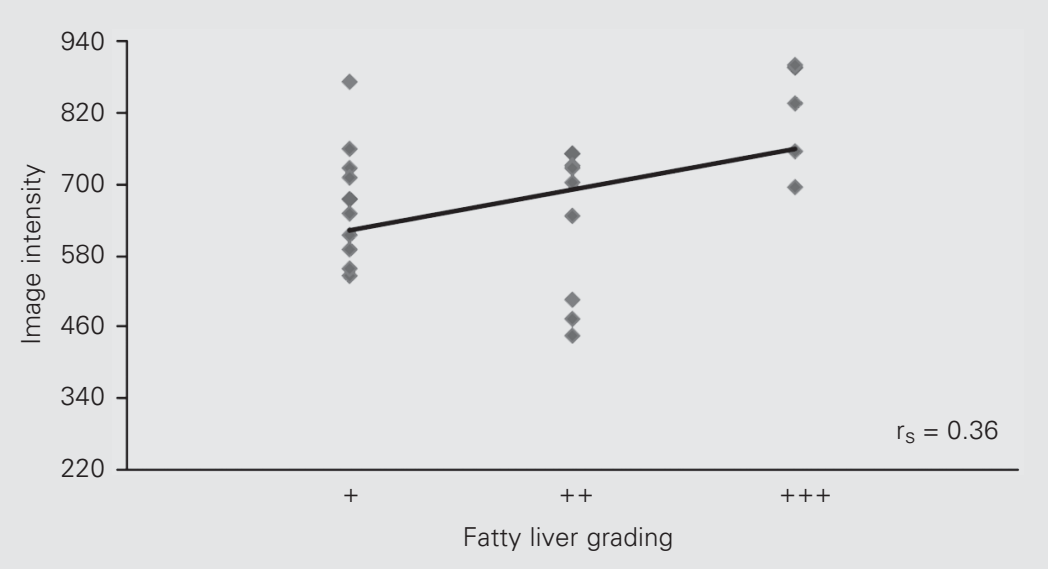

Figure 1. Correlation between conventional magnetic resonance imaging and the histological grading of hepatic steatosis. Fatty liver was graded as described in Ref. 14: +, up to $33 \% ;++, 33-66 \% ;+++,>66 \%$.

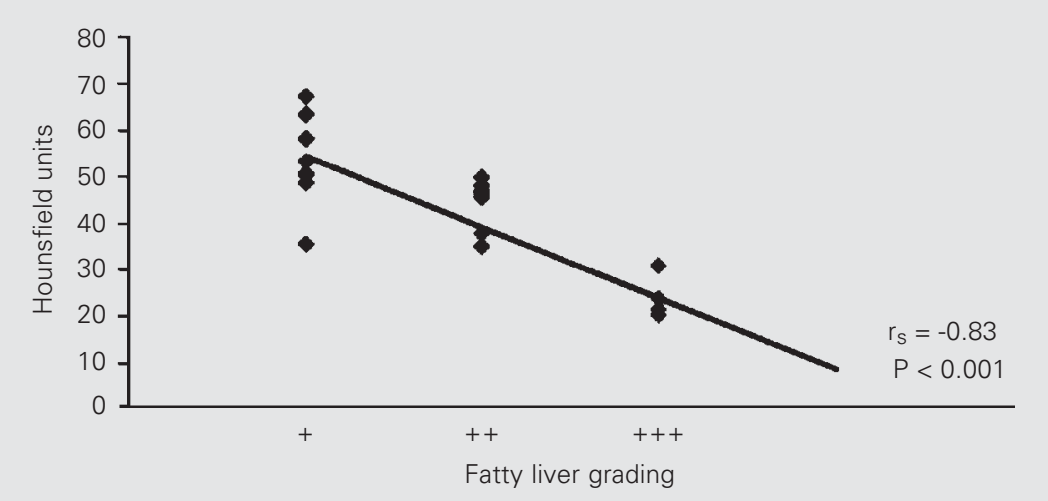

Figure 2. Correlation between computed tomography attenuation index and the histological grading of hepatic steatosis. Fatty liver was graded as described in Ref. 14: +, up to $33 \% ;++, 33-66 \% ;+++,>66 \%$. 
mained stable (treated group, BMI before $v s$ end of treatment $=30 \pm 0.7$ vs $29.8 \pm 0.9$; $\mathrm{P}$ $=0.893$ ).

Two patients (one from each group) were excluded because of lack of compliance with treatment. No patient presented side effects that could be attributed to the use of UDCA.

In the placebo group no significant modifications in mean AST, ALT or $\gamma$-GT values were observed throughout the study. In contrast, a significant reduction in the mean values for these enzymes was observed in the UDCA group, especially during the first two months and also in the third month for ALT (Figure 3). The response did not differ in terms of degree of obesity or type of NAFLD.

No differences were observed between the values of hepatic density obtained at the beginning and at the end of the study (treated group $=50.2 \pm 4.2$ vs $51.1 \pm 4.1$; placebo group $=48.6 \pm 5.0 v s 48.1 \pm 5.1)$.

\section{Discussion}

It is now clear that the obese patient can develop NAFLD, with hepatic inflammation, fibrosis and even cirrhosis $(16,17)$. According to many investigators, fatty liver, mainly with elevated serum liver enzymes, can progress to NASH and in some to cirrho-

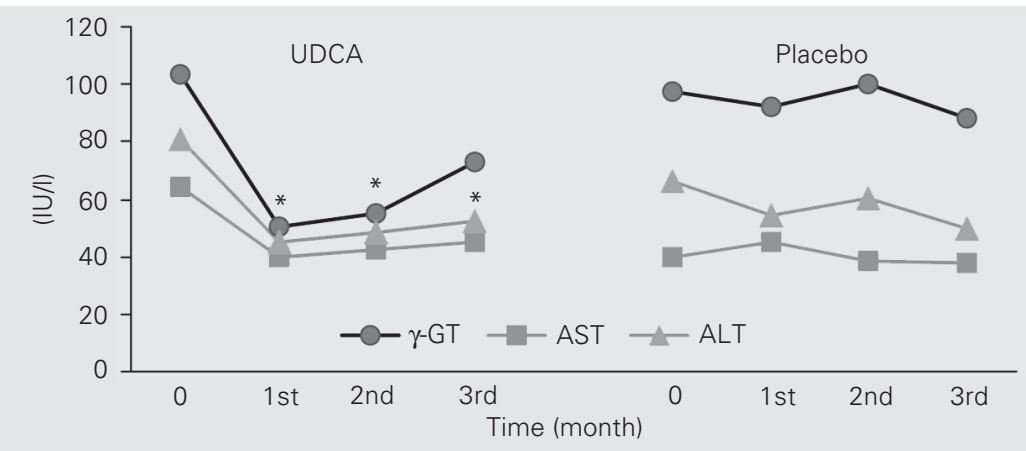

Figure 3. $\gamma$-Glutamyltransferase $(\boldsymbol{\gamma}$-GT), aspartate (AST) and alanine (ALT) aminotransferase values of the ursodeoxycholic acid (UDCA)-treated and untreated (placebo) patients determined at the beginning and after the first, second and third month of the experiment. Data are reported for 14 patients in each group. Error bars were omitted to improve the understanding of the figure. ${ }^{*} \mathrm{P}<0.05$ compared to the beginning of the experiment (Bonferroni test). sis (about 7-26\%). NAFLD can be present in up to $20 \%$ of obese patients and in 3 to $4 \%$ of those with normal weight, reaching an overall prevalence of $13 \%(1,14,16-18)$. Early studies demonstrated a higher prevalence among women, related to the higher incidence of obesity in women or secondary to hormonal effects; however, it is now known that nonobese men are equally affected $(16,19,20)$. These differences, however, seem to depend basically on how the patients are selected for the study. In the present study, patients were selected on the basis of abnormal ALT detected during blood donation.

All patients had NAFLD associated with obesity with or without associated dyslipidemia or diabetes. Since these patients had stable obesity in the last months and were studied for a short period of time, BMI becomes a parameter of easy control to assess whether modifications in body mass are interfering with the patient's treatment with UDCA.

Increases in serum ALT, AST and $\gamma$-GT are frequently observed in NAFLD, although their levels are often poorly correlated with histological parameters $(1,14,19)$.

For a proper image analysis, patient weight was limited to $120 \mathrm{~kg}$ since higher values interfere with the study of the correlation between the degree of obesity and the intensity of steatosis reported by others $(21,22)$.

There is evidence of an inverse correlation between $\mathrm{CT}$ attenuation in the liver and the hepatic degree of steatosis (4). Thus, it has been suggested that $\mathrm{CT}$ might provide an alternative method for quantitatively estimating liver fat content. Absolute liver attenuation values are considered to be of limited use because they can vary depending on technical variables like scanner type, tube current, as well as individual variations in patient conditions such as cardiac or renal function (23). We believe that these limitations will be reduced with new generation scanners. 
MRI has been considered to be the gold standard for the noninvasive quantification of fat content in the liver when the technique of Dixon is used $(3,24)$. In the present study we found traditional MRI to be less valuable than CT for the quantification of NAFLD. In fact, a significant correlation of $\mathrm{CT}$ with the degree of histological steatosis confirmed results of previous and recently published studies $(4,25)$.

It has already been stated that the basic treatment of patients with obesity related to NAFLD would be weight loss. It is observed, however, that most of these individuals are not able to reach this objective in a consistent way, needing a more effective treatment $(5,12,26)$. In studies of isolated cases $(11)$ or in uncontrolled studies (12) a histological and biochemical improvement of NAFLD was observed after one year of treatment with UDCA. However, since those were not controlled studies, and most patients were obese and were strongly encouraged to lose weight, it is difficult to attribute the observed improvements exclusively to the medication. More recently, it was shown that patients with NASH (27) and NAFLD (28) treated with a low fat diet associated with UDCA presented a more pronounced improvement than those treated with diet only. But again, the effects of UDCA could have been masked by the diet.

In the present study patients were randomized in a double-blind manner to use UDCA or placebo for three months based on studies that showed a significant reduction or disappearance of steatosis due to nutritional deficiencies during this period of time $(29,30)$. The daily dose of $10 \mathrm{mg} \mathrm{kg}^{-1} \mathrm{day}^{-1}$ was chosen on the basis of previous studies with UDCA for the treatment of cholelithiasis and hepatic cholestatic diseases $(7,8,10$, 29), and also because lower doses for obese patients substantially reduce the cost of treatment.

During treatment, no significant alterations were found in the mean values of
AST, ALT or $\gamma$-GT in the control group, while in the UDCA-treated group a statistically significant fall in these parameters was demonstrable during the first two months and up to the third month for ALT. These results demonstrate a positive effect of UDCA by reducing hepatic enzyme levels in patients with NAFLD, confirming previously published results $(11,12,27,28)$.

Several reports have confirmed that UDCA improves the hepatic tests in patients with chronic hepatic disease $(10,26)$. However, the exact mechanism of this action is not understood. The drug may alter intestinal absorption (31) or eliminate toxic hydrophobic bile acids from the liver or reduce the toxic effects of these hydrophobic compounds on hepatocyte membranes $(32,33)$. UDCA may also reduce expression of class II HLA inhibitors and interleukin and interferon production, acting as an immunomodulator (34), in addition to having the potential to reduce reactive oxygen species $(35,36)$.

In obese patients with NAFLD, triglyceride accumulation in the liver is due to an increased mobilization and availability of free fatty acids from peripheral adipose tissues, increased hepatic free fatty acids synthesis and esterification and decreased export of triglycerides from the liver $(37,38)$. Oxidative stress or endotoxic cytokine-mediated injury may follow this fat accumulation, leading to hepatic fibrosis and cirrhosis $(17,30,39)$.

When CT was used as a method of quantification of the lipid content of the liver, no significant differences were found between results obtained at the beginning and at the end of treatment in either group. Since this evaluation was only performed again at the end of the experiment, we may have missed some modifications in this parameter during the first two months of follow-up when the biochemical response was maximal. However, due to the short time of follow-up and to the fact that ALT levels were still significantly reduced when CT was performed, it is 
more likely that such enzymatic alterations observed in the treated group were not related to a reduction in hepatic lipid content.

Under these conditions, improvement in hepatic enzyme levels in NAFLD could be related to fat reduction in cell and mitochondrial membranes secondary to hydrophobic toxic bile acids $(32,33,40)$. It is also possible that UDCA could reduce oxidative stress in NAFLD due to an increase in hepatocyte levels of glutathione and thiol-containing proteins, as proposed by others (35).

The results of the present study suggest that a CT scan is useful for the assessment of the degree of liver steatosis and demonstrate a transitory and significant effect of UDCA on the reduction of hepatic enzyme levels in patients with NAFLD. Such effect is not related to modifications in liver fat content, or in BMI. The exact mechanism by which UDCA acts on NAFLD remains to be established. It also remains to be ascertained whether a sustained effect could be obtained with higher and prolonged doses of UDCA, as used by others (12).

\section{Acknowledgments}

Ursodeoxycholic acid was kindly provided by Zambon Laboratories, São Paulo, SP, Brazil.

\section{References}

1. Matteoni CA, Younossi ZM, Gramlich T, Boparai N, Liu YC \& McCullough AJ (1999). Nonalcoholic fatty liver disease: a spectrum of clinical and pathological severity. Gastroenterology, 116: 14131419.

2. Ratziu V, Giral P, Charlotte F, Bruckert E, Thibault V, Theodorou I, Khalil L, Turpin G \& Opolon P (2000). Liver fibrosis in overweight patients. Gastroenterology, 118: 1117-1123.

3. Saadeh S, Younossi ZM, Remer EM, Gramlich T, Ong JP, Hurley M, Mullen KD, Cooper JN \& Sheridan MJ (2002). The utility of radiological imaging in nonalcoholic fatty liver disease. Gastroenterology, 123: 745-750.

4. Ricci C, Longo R, Gioulis E et al. (1997). Obesity-related nonalcoholic fatty liver: CT features and follow-up studies after low-calorie diet. Radiology, 162: 845-847.

5. Palmer M \& Schaffner $F$ (1990). Effect of weight reduction on hepatic abnormalities in overweight patients. Gastroenterology, 99: 1408-1411.

6. Ueno T, Sugawara H, Sujaku K, Hashimoto O, Tsuji R, Tamaki S, Torimura T, Inuzuka S, Sata M \& Tanikawa S (1997). Therapeutic effects of restricted diet and exercise in obese patients with fatty liver. Journal of Hepatology, 27: 103-107.

7. Beuers U, Spengler U, Kruis W, Aydemir Ü, Wiebecke B, Heldwein W, Weinzierl M, Pape GR, Sauerbruch T \& Paumgartner G (1992). Ursodeoxycholic acid for treatment of primary sclerosing cholangitis: a placebo-controlled trial. Hepatology, 16: 707-714.

8. Crosignani A, Battezzati PM, Setchell DR, Camisasca M, Bertolini E, Roda A, Zuim N \& Podda M (1991). Effects of ursodeoxycholic acid metabolism in chronic active hepatitis: a dose-response study. Hepatology, 13: 339-344

9. Plevris JN, Hayes PC \& Bouchier AD (1991). Ursodeoxycholic acid in the treatment of alcoholic liver disease. European Journal of Gastroenterology and Hepatology, 3: 653-656.

10. Poupon RE, Balkau B, Eschwege E \& Poupon R (1991). A multicenter, controlled trial of ursodiol for the treatment of primary biliary cirrhosis. New England Journal of Medicine, 324: 1548-1554.

11. Abdelmalek M, Ludwig J \& Lindor KD (1995). Two cases from the spectrum of nonalcoholic steatohepatitis. Journal of Clinical Gastroenterology, 20: 127-130

12. Laurin J, Lindor KD, Crippin JS, Gossard A, Gores GJ, Ludwig J Rakela J \& McGill DB (1996). Ursodeoxycholic acid or clofibrate in the treatment of non-alcohol-induced steatohepatitis: a pilot study. Hepatology, 23: 1464-1467.

13. Garrow JS \& Webster J (1985). Quetelet's index (W/H2) as measure of fatness. International Journal of Obesity, 9: 147-153.

14. Teli MR, James OFW, Burt AD, Bennett MK \& Day CP (1995). The natural history of nonalcoholic fatty liver: A follow-up study. Hepatology, 22: 1714-1719.

15. Glantz AG (1992). Primer of Bio-Statistics. 3rd edn. McGraw-Hill, Inc., New York.

16. Ludwig J, Viggiano TR, McGill DB \& Ott BJ (1980). Nonalcoholic steatohepatitis. Mayo Clinic experiences with a hitherto unnamed disease. Mayo Clinic Proceedings, 55: 434-438.

17. Neuschwander-Tetri BA (2002). Evolving pathophysiologic concepts in nonalcoholic steatohepatitis. Current Gastroenterology Reports, 4: 31-36.

18. Wanless IR \& Lentz JS (1990). Fatty liver hepatitis (steatohepatitis) and obesity: an autopsy study with analysis of risk factors. Hepatology, 12: 1106-1110.

19. Angulo P (2002). Nonalcoholic fatty liver disease. New England Journal of Medicine, 346: 1221-1231.

20. Clark JM, Brancati FL \& Diehl AE (2001). Nonalcoholic fatty liver disease: the most common cause of abnormal liver enzymes in the US population. Gastroenterology, 120: A-65 (Abstract).

21. Andersen T \& Gluud C (1984). Liver morphology in morbid obesity: a literature study. International Journal of Obesity, 8: 97-106.

22. Kern WH, Heger AH, Payne JH \& DeWind LT (1973). Fatty metamorphosis of the liver in morbid obesity. Archives of Pathology, 96: 342-346.

23. Ishikara I, Tateishi K \& Shinoda A (1990). Changes of the hepatic CT absorption value in hemodialysis patients. Journal of Computer Assisted Tomography, 14: 1013-1015.

24. Dixon WT (1984). Simple proton spectroscopic imaging. Radiology, 
153: 189-194.

25. Shigueoka DC (1996). Distribuição dos coeficientes de atenuação do fígado no estudo da esteatose pela tomografia computadorizada. Master's thesis, Universidade Federal de São Paulo, São Paulo, SP, Brazil.

26. Leuschner U, Leuschner M, Sieratzki J, Kurtz W \& Hübner K (1985). Gallstone dissolution with ursodeoxycholic acid in patients with chronic active hepatitis and two years follow-up. A pilot study. Digestive Diseases and Sciences, 30: 642-649.

27. Guma G, Viola L, Thome M, Gadame O \& Alvarez E (1997). Ursodeoxycholic acid in the treatment of non-alcoholic steatohepatitis: results of a prospective clinical controlled trial. Hepatology, 26 (Part 2): 387A (Abstract).

28. Ceriani R, Brunati S, Morini L, Sacchi E \& Colombo G (1998). Effect of ursodeoxycholic acid plus diet in patients with non-alcoholic steatohepatitis. Hepatology, 28 (Part 2): 386A (Abstract).

29. Doherty JF, Adam EJ, Griffin GE \& Golden MHN (1992). Ultrasonographic assessment of the extent of hepatic steatosis in severe malnutrition. Archives of Disease in Childhood, 67: 1348-1352.

30. Eriksson S, Eriksson K \& Bondesson L (1986). Nonalcoholic steatohepatitis in obesity: a reversible condition. Acta Medica Scandinavica, 220: 83-88.

31. Marteau P, Chazouilléres O, Myara A, Jian R, Rambaud JC \& Poupon $R$ (1990). Effect of chronic administration of ursodeoxycholic acid on the ileal absorption of endogenous bile acids in man. Gastroen- terology, 12: 1206-1208.

32. Palmer $\mathrm{RH}$ (1972). Bile acids, liver injury, and liver disease. Archives of Internal Medicine, 130: 606-615.

33. Queneau PE \& Montet JC (1994). Hepatoprotection by hydrophilic bile salts. Journal of Hepatology, 21: 260-268.

34. Calmus Y, Gane P, Rouger P \& Poupon R (1990). Hepatic expression of class I and class II major histocompatibility complex molecules in primary biliary cirrhosis: effect of ursodeoxycholic acid. Hepatology, 11: 12-15.

35. Mitsuyoshi H, Nakashima T, Sumida Y, Yoh T \& Nakajima Y (1999). Ursodeoxycholic acid protects hepatocytes against oxidative injury via induction of antioxidants. Biochemical and Biophysical Research Communications, 263: 537-542.

36. Rodrigues CM, Fan Wong PY, Kren BT \& Sterr CJ (1998). Ursodeoxycholic acid may inhibit deoxycholic acid induced apoptosis by modulation of mitochondrial transmembrane potential and reactive oxygen species production. Molecular Medicine, 4: 165-178.

37. Day CP \& James OFW (1998). Steatohepatitis: a tale of two "hits"? Gastroenterology, 114: 842-845.

38. Younossi ZM (1999). Non-alcoholic fatty liver disease. Current Gastroenterology Reports, 1: 57-61.

39. Younossi ZM, Diehl AM \& Ong JP (2002). Nonalcoholic fatty liver disease: an agenda for clinical research. Hepatology, 35: 746-752.

40. Heuman DM (1993). Hepatoprotective properties of ursodeoxycholic acid. Gastroenterology, 104: 1865-1869. 